\title{
Flavonoids furom Coreopsis tinctoria adjust lipid metabolism in hyperlipidemia animals by down-regulating adipose differentiation-related protein
}

Yali Li ${ }^{1 \dagger}$, Xinmei Chen ${ }^{2 \dagger}$, Jie Xue ${ }^{3}$, Jiangyun Liư ${ }^{3}$, Xinhua Chen ${ }^{4^{*}}$ and Muhuyati Wulasihan ${ }^{{ }^{*}}$

\begin{abstract}
Background: To identify the chemical structure of Coreopsis tinctoria extracts and their effect and mechanism on reducing blood lipid in hyperlipemia mice.

Methods: The flavonoids were extracted from Coreopsis tinctoria. The chemical structure was identified by HPLC. 59 mice were divided randomly into 5 groups. (group 1: normal diet control; group 2: hyperlipemia model; group 3: hyperlipemia mice treated with Coreopsis tinctoria, low dose $100 \mathrm{mg} / \mathrm{kg}$; group 4: hyperlipemia mice treated with Coreopsis tinctoria high dose group 200 mg/kg; group 5 hyperlipemia mice treated with Fenofibrate. After 2 week of hyperlipid diet, the treatment of Coreopsis tinctoria and Fenofibrate were given for another 6 weeks with continuous hyperlipid diet. The TC, TG, HDL, histology, adipose differentiation-related protein (ADRP) expression in different groups were compared.
\end{abstract}

Results: Compared with normal diet group, TC, TG in hyperlipemia model group increased $(P<0.01)$. After treatment with Coreopsis tinctoria low dose group, high dose group, TC of the hyperlipemia mice decreased $(P<0.05)$ without increasing AST, ALT and ALP. Fenofibrate can also decrease TC and TG but increase AST, ALT and ALP. Expression of hepatic ADRP increased in hyperlipemia mice. Coreopsis tinctoria high dose group $200 \mathrm{mg} / \mathrm{kg}$ can inhibit ADRP as Fenofibrate does.

Conclusion: The flavonoids from Coreopsis tinctoria extracts can reduce blood lipid without liver function damage, showing better anti- hyperlipemia effect than Fenofibrate by down-regulating ADRP.

Keywords: Coreopsis tinctoria, Flavonoid, Hyperlipemia, Fenofibrate, Adipose differentiation-related protein (ADRP)

\section{Introduction}

Hyperlipidemia is clinically common disease with the rapid improvement of economy and quick changes in diet. The incidence of hyperlipidemia is threatening people's health. A number of studies indicate that hyperlipidemia is an independent risk factor for stroke, coronary heart disease and sudden cardiac death, and is closely related to the

\footnotetext{
*Correspondence: xinhua_chen@zju.edu.cn; muhuyati@163.com

${ }^{\dagger}$ Equal contributors

${ }^{4}$ The Department of Hepatobiliary and Pancreatic Surgery, The First Affiliated Hospital Zhejiang University School of Medicine, Hangzhou, Zhejiang 310003, China

${ }^{1}$ The Department of Internal Medicine, The First Affiliated Hospital of Xinjiang Medical University Urumqi, Xinjiang 830000, China

Full list of author information is available at the end of the article
}

occurrence of non-alcoholic fatty liver disease [1]. Fenofibrate helps reduce cholesterol and triglycerides in the blood to increase risk of atherosclerosis but the side effect on liver function damage are not rare [2]. Therefore, effectively and safe regulation of blood lipids and cholesterol is significant for prevention and treatment. Botanicals extract is rich of flavonoids and recent research show it plays an important role in blood lipid adjustment [3].

Coreopsis tinctoria in Xinjiang is also known as Xinjiang chrysanthemum in Snow Mountain [4]. It has multiple pharmaceutical functions such as heat-clearing, detoxifying, blood circulation promoting and blood stasis removing. Local Uyghur people use it as an herb tea to treat high blood pressure and diarrhea. Modern

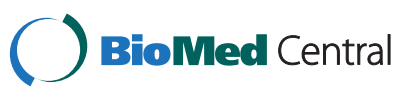


pharmacological studies have shown that it can adjust blood sugar, blood lipid and blood pressure $[5,6]$. It is reported that Coreopsis tinctoria in Xinjiang is rich of flavonoids and can adjust glucose metabolism and reduce cardiovascular disease risk $[5,6]$. There has not research on lipid metabolism [7]. In this study we investigate its active ingredient identification, biomedical function and molecular mechanism on lipid metabolism.

\section{Material and methods}

\section{Instruments}

Electronic scale (Mettler-Toledo manufacturing company, Model AL104). Homogeneous dispersion machine (Shanghai Jinda biochemistry Instrument CO. Model FJ-200). Spectrophotometer (Kodak, Model 722). Visible Spectrophotometer (Shanghai Scientific Instrument Company, Model $722 \mathrm{~N})$. High lipid diet was made of lard which was purchased commercially in local market. Cholesterol was from HuiXing, China Biochemical Reagents LLT, analytical grade, LOT number 120312. Sucrose was provided by the Yancheng Honey Garden Food CO, LOT number 20120915). Propyl thiouracil was provided by Shanghai ZhaoHui Pharmaceutical, LOT number 20120206). Propylene glycol and polysorbate- 80 was provided by Reed Sinopharm Chemical Company, analytical grade, LOT number T20110418 and F20110726, respectively. Total cholesterol (TC), triglyceride (TG) and high-density lipoprotein cholesterol (HDL-c), low-density lipoprotein cholesterol (LDL-c) and alanine aminotransferase (ALT), aspartate aminotransferase (AST), alkaline phosphatase (ALP) kits are provided by Beijing Beihua Kangtai Clinical Reagent, LOT number: 20130717). Fenofibrate was used as a positive control and mixed with $0.5 \% \mathrm{CMC}-\mathrm{Na} 2$ to make $0.2 \%$ solution. Drugs were sealed and placed in refrigerator for the further tests.

\section{Process of flavonoids extract}

Coreopsis tinctoria were collected from Dabanchen Xinjiang, China (LOT number 130501) and the botanical origin of material was identified by Prof. Xinmei Chen, College of Pharmaceutical Sciences, Shandong Traditional Medical University, Jinan, China. Dry flowers (20 kg) were extracted with $70 \%$ aqueous ethanol (v/v) for two times (200 L, $1.5 \mathrm{~h}$ each) under reflux. After evaporation, the residue was suspended in $\mathrm{H} 2 \mathrm{O}(10 \mathrm{~L})$ and filtrated, the liquor solution was then subjected to $\mathrm{AB}-8$ macroporous resin column $(80 \times 10 \mathrm{~cm}$ i.d.), eluting with $\mathrm{H} 2 \mathrm{O}, 20 \%, 70 \%, 95 \%$ ethanol (each $30 \mathrm{~L}$ ) successively. The $70 \%$ and $95 \%$ eluants were combined and concentrated under reduced pressure to give the total flavonoid extract (PME, $114.5 \mathrm{~g}$ ), which was applied to the following test in this study.

\section{HPLC analysis}

The HPLC system consisted of a two solvent delivery system (LC-20AB), a vacuum degasser, a column thermostat
(CTO-20A), a photodiode array detector and LC-Solution (Shimadzu Corporation, Japan). Detection wavelengths were set at $330 \mathrm{~nm}$. A Cosmosil C18 AR - П column $(250 \mathrm{~mm} \times 4.6 \mathrm{~mm}, 5 \mu \mathrm{m})$ was used with a flow rate of $1.0 \mathrm{~mL} \cdot \mathrm{min}-1$. The injection volume was $10 \mu \mathrm{L}$ and the column temperature was maintained at $35^{\circ} \mathrm{C}$. An isocratic elution was adopted with $14 \%$ acetonitrile- $0.1 \%$ acetic acid as mobile phase.

\section{Animal experiments for hyperlipidemia animal model}

A total of 59 male mice $(20 \pm 2$ g) were acquired from the Zhaoyan Laboratory Animal Co., Ltd. (Soochou, China). All experiments were performed in compliance with the Chinese Legislation on The Use And Care of Laboratory Animals and were approved by the committee on animal care and use of Soochow University (Laboratory animal production license: SCXK 2013-0003, laboratory animal use license: SYXK 2012-0045). Animals were maintained in Soochow University School of Medicine laboratory animal Center. Room temperature $20 \pm 2^{\circ} \mathrm{C}$, humidity 55-65\%, 12 h/12 h light-dark cycle with free feeding and drinking water.

The non-hyperlipidemia control group were given normal diet $(n=11)$; hyperlipidemia anima model was induced in the mice by oral feeding of high fat diet (lard 20\%, cholesterol $10 \%$, bile salts $2 \%$, Propylthiouracil $0.2 \%$, propylene glycol $20 \%$, polysorbate- $8020 \% .0 .2 \mathrm{mg} /$ per $10 \mathrm{~g}$ weight per day) for 2 weeks. The hyperlipidemia animal model was confirmed by blood lipid test and then the confirmed hyperlipidemia mice were randomly divided into 4 groups. With continuous high fat diet, the following treatments were administered for another 6 weeks: Hyperlipidemia group $(n=12)$, the low-dose group (oral feeding of Coreopsis tinctoria $100 \mathrm{mg} / \mathrm{kg}, \mathrm{n}=12$ ), high-dose groups (oral feeding of Coreopsis tinctoria $200 \mathrm{mg} / \mathrm{kg}, \mathrm{n}=12$ ), Fenofibrate group (oral feeding of Fenofibrate $40 \mathrm{mg} / \mathrm{kg}$, $\mathrm{n}=12$ ). After the final treatment, the mice were weighed and then sacrificed. All liver specimens in each group were fixed with $10 \%$ buffered formalin and embedded in paraffin for slicing after weighing. Five sections in each group were used for histopathology examination and others for Immunohistochemical examination. Sections were stained with hematoxylin and eosin for histopathology examination. The blood sample was taken for blood lipid and liver function test.

\section{Blood lipid and liver function test}

The serum TG, TC, LDL, HDL, ALT, AST and ALP were determined during the treatment period by serum TC and TG once every 2 weeks. After final treatment, the mice were given no food for $12 \mathrm{~h}$. Blood sample was taken from orbital vein, centrifuge $15 \mathrm{~min}$ in $3500 \mathrm{r} / \mathrm{min}$ to get its serum for TC, TG, HDL, LDL, AST and ALT measurement. After collecting blood in mice, the liver 

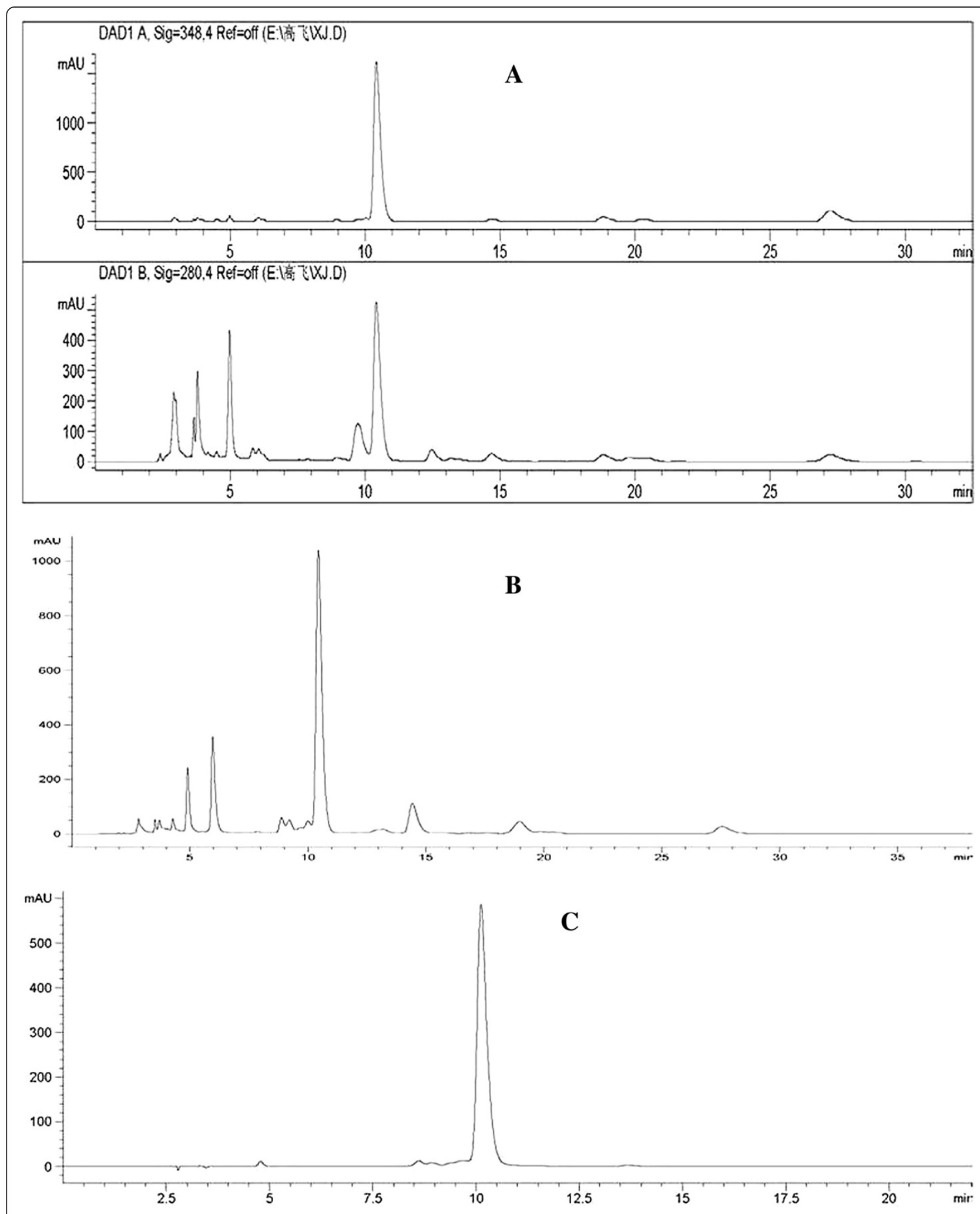

Figure 1 HPLC/DAD analysis of extract from Xinjiang Coreopsis tinctoria. HPLC chromatographic profile of Coreopsis tinctoria extracts. (A) Total Coreopsis tinctoria extracts from dry flower top (B) total flavonoid at $348 \mathrm{~nm}$. (C) the major compound marinin at $348 \mathrm{~nm}$. 
Table 1 Average food intake changes (g/mice)

\begin{tabular}{lccccc}
\hline & Normal diet $(\mathbf{n}=\mathbf{1 1})$ & Hyperlipidemia $(\mathbf{n}=\mathbf{1 2})$ & $\mathbf{C T} \mathbf{1 0 0} \mathbf{~} \mathbf{~ g} / \mathbf{k g}(\mathbf{n}=\mathbf{1 2})$ & $\mathbf{C T} \mathbf{2 0 0} \mathbf{~ m g} / \mathbf{k g}(\mathbf{n}=\mathbf{1 2})$ & Fenofibrate $(\mathbf{n}=\mathbf{1 2})$ \\
\hline High lipid diet 1 week & $6.19 \pm 0.46^{* *}$ & $5.36 \pm 0.22$ & $5.30 \pm 0.25$ & $5.30 \pm 0.22$ & $5.33 \pm 0.23$ \\
High lipid diet 2 weeks & $6.30 \pm 0.89^{* *}$ & $4.91 \pm 0.58$ & $5.12 \pm 0.55$ & $5.06 \pm 0.57$ & $4.94 \pm 0.35$ \\
Treatment 1 week & $6.58 \pm 1.43$ & $5.22 \pm 1.16$ & $5.56 \pm 1.19$ & $5.25 \pm 1.23$ & $5.62 \pm 1.08$ \\
Treatment 2 weeks & $4.62 \pm 0.18$ & $4.61 \pm 0.29$ & $4.55 \pm 0.19$ & $4.45 \pm 0.24$ & $4.57 \pm 0.26$ \\
Treatment 3 weeks & $5.01 \pm 0.02$ & $4.55 \pm 0.47$ & $4.56 \pm 0.28$ & $4.20 \pm 0.46$ & $4.32 \pm 0.40$ \\
Treatment 4 weeks & $4.77 \pm 0.22$ & $4.30 \pm 0.61$ & $4.28 \pm 0.35$ & $4.20 \pm 0.49$ & $4.20 \pm 0.33$ \\
Treatment 5 weeks & $4.56 \pm 0.03^{* *}$ & $3.97 \pm 0.22$ & $4.18 \pm 0.19$ & $3.99 \pm 0.19$ & $3.99 \pm 0.27$ \\
Treatment 6 weeks & $4.57 \pm 0.02^{* *}$ & $3.95 \pm 0.16$ & $4.12 \pm 0.23$ & $3.94 \pm 0.23$ & $4.02 \pm 0.13$ \\
\hline
\end{tabular}

**P $<0.01$ vs model group.

was quickly removed and scaled for calculating index of liver (liver weight index $=$ hepatic mass/body mass $\times 100 \%$ ). The left lobe of the liver, was grounded at $3000 \mathrm{r} / \mathrm{min}$ in centrifuge for $10 \mathrm{~min}$ to get the supernatant for TC, TG measurement. The rest of hepatic tissues were snap-frozen in liquid nitrogen and stored at $-80^{\circ} \mathrm{C}$ for Western blot.

\section{Morphological evaluation}

Liver index was obtained by dividing liver weight by mouse weight and then times $100 \%$. The liver specimens were fixed with $10 \%$ neutral formalin and embedded in paraffin. Paraffin sections of liver were stained with H\&E for histopathological examination. Fat accumulation in liver. Was graded according to the methods (Bujanda etal., 2008; QinandTian, 2010)(grade0: no fat was found; grade1: less than 33\%; grade2:33-66\%; grade3:more than $66 \%$ of hepatocytes were affected by fatvacuoles).

\section{Western blotting analysis}

Hepatic tissue samples protein concentration was determined using a BCA Kit (Pierce, Rockford, IL, USA). Samples containing $50 \mu \mathrm{g}$ of protein each were loaded on a $10 \%$ SDS-PAGE gel and then transferred to a nitrocellulose membrane. The membrane was then rinsed and incubated with witha1:1000 dilution of a rabbit polyclonalanti mouse ADRP (Santa Cruz Biotechnology, California, USA) or anti GAPDH (1:5000 dilution) (Sigma, Missouri, USA).
Membranes were then washed and incubated with horseradish-peroxidase-conjugated donkey anti-mouse IgG at 1:5000. The ECL ${ }^{\mathrm{Tm}}$ Western blotting detection reagent (Amersham Biosciences, New Jersey, USA) was used for visualization and the results were analyzed quantitatively using SigmaScan Pro 5.0. The data were normalized with respect to ratios of GAPDH detected on the same blot to control for variation in protein loading across samples.

\section{Statistical analysis}

All data are expressed as means \pm SD. The significance of difference among groups was determined by using one-way ANOVA. $\mathrm{P}<0.05$ was considered statistically significant.

\section{Results}

Identification of flavonoids in Coreopsis tinctoria

Marein, $0.8653 \mathrm{mg} / \mathrm{Ml}$ was used as standard, using HPLC/ PDA system to establish a qualitative and quantitative analysis of herbs and total flavonoids. Chromatographic conditions: Shimadzu LC 20A HPLC liquid systems, Cosmosil C18 column $(4.6 \times 250 \mathrm{~mm})$, acetonitrile $-0.1 \%$ ammonium acetate (20:80) as the mobile phase, flow rate $1.0 \mathrm{ml} /$ min, injection volume $10 \mathrm{ul}$; PDA detection wavelength: $280,348 \mathrm{~nm}$. The results shown in Figure 1. The HPLC conditions chosen were adequate to separate compounds as shown in chromatograms presented in Figure 1. The

Table 2 The average mouse weight change during the experiment (g/mice)

\begin{tabular}{llccccc}
\hline Week & Treatment & Normal diet $(\mathbf{n}=\mathbf{1 1})$ & Hyperlipidemia $(\mathbf{n}=\mathbf{1 2})$ & $\mathbf{C T} \mathbf{1 0 0} \mathbf{~ m g} / \mathbf{k g}(\mathbf{n}=\mathbf{1 2})$ & $\mathbf{C T ~ 2 0 0} \mathbf{m g} / \mathbf{k g}(\mathbf{n}=\mathbf{1 2})$ & Fenofibrate $(\mathbf{n}=\mathbf{1 2})$ \\
\hline 0 & Begin & $24.48 \pm 1.80$ & $24.03 \pm 2.26$ & $24.13 \pm 2.12$ & $24.21 \pm 1.94$ & $23.53 \pm 2.06$ \\
1 & High lipid diet & $32.69 \pm 1.91$ & $31.96 \pm 2.11$ & $32.19 \pm 1.50$ & $31.56 \pm 1.41$ & $32.10 \pm 1.88$ \\
2 & High lipid diet & $36.93 \pm 1.81$ & $35.43 \pm 2.39$ & $36.22 \pm 2.02$ & $35.11 \pm 2.46$ & $35.81 \pm 2.75$ \\
3 & treatment & $40.37 \pm 1.89$ & $38.33 \pm 2.89$ & $39.06 \pm 2.11$ & $38.16 \pm 2.11$ & $37.94 \pm 3.35$ \\
4 & treatment & $42.05 \pm 2.31$ & $39.98 \pm 3.01$ & $40.61 \pm 2.45$ & $39.45 \pm 1.82$ & $39.82 \pm 3.47$ \\
5 & treatment & $44.11 \pm 2.41$ & $43.40 \pm 3.49$ & $43.10 \pm 2.85$ & $42.42 \pm 2.22$ & $42.03 \pm 4.05$ \\
6 & treatment & $45.77 \pm 2.39$ & $44.33 \pm 3.33$ & $43.87 \pm 2.53$ & $43.71 \pm 2.22$ & $43.07 \pm 4.35$ \\
7 & treatment & $47.07 \pm 2.88$ & $45.38 \pm 3.47$ & $45.66 \pm 2.79$ & $44.97 \pm 2.37$ & $44.22 \pm 4.17$ \\
8 & treatment & $48.61 \pm 3.06$ & $46.92 \pm 3.82$ & $47.31 \pm 3.02$ & $46.83 \pm 2.47$ & $46.16 \pm 4.77$ \\
\hline
\end{tabular}


Table 3 The blood lipid levels at 2 weeks and 4 weeks after drug administration

\begin{tabular}{|c|c|c|c|c|c|}
\hline & \multirow[t]{2}{*}{$n$} & \multicolumn{2}{|c|}{$\mathrm{TC}(\mathrm{mmol} / \mathrm{L})$} & \multicolumn{2}{|c|}{ TG (mmol/L) } \\
\hline & & 2 week & 4 week & 2 week & 4 week \\
\hline Normal diet & 11 & $3.4 \pm 0.43^{* *}$ & $3.28 \pm 0.39^{* *}$ & $1.56 \pm 0.38^{* *}$ & $2.19 \pm 0.54^{*}$ \\
\hline Model & 12 & $4.75 \pm 0.69$ & $4.53 \pm 0.60$ & $2.96 \pm 0.96$ & $2.83 \pm 0.76$ \\
\hline $\mathrm{CT} 100 \mathrm{mg} / \mathrm{kg}$ & 12 & $4.39 \pm 0.63$ & $4.14 \pm 0.48$ & $2.24 \pm 0.91$ & $2.04 \pm 0.68^{*}$ \\
\hline CT $200 \mathrm{mg} / \mathrm{kg}$ & 12 & $4.29 \pm 0.48$ & $4.02 \pm 0.38^{*}$ & $2.12 \pm 0.55^{*}$ & $2.18 \pm 0.68 *$ \\
\hline Fenofibrate & 12 & $4.05 \pm 0.84^{*}$ & $3.70 \pm 0.68^{* *}$ & $2.72 \pm 0.97$ & $2.23 \pm 0.48^{*}$ \\
\hline
\end{tabular}

Compared with hyperlipidemia model, ${ }^{*} \mathrm{P}<0.05$;**P $<0.01$.

photodiode array detection (DAD) in tandem with electron spray ionization (ESI) mass spectrometry provided chemical composition of the fraction and they were mainly aglycones and glycosides of flavanone/chalcone flavonoid type. Compound marein appears as the major peak detected. The major 12 compounds seperated from LC/PDA/ESIMS chromatogram include 3,4,5,6,7-Pentahydroxyflavanone-O-hexoside, Chlorogenic Acid, Flavanomarein, Flavanokanin, Quercetagitin-7-O-glucoside, 3,4', 5,6,7-Pentahydroxyflavanone, 3',5,5',7-Tetrahydroxyflavanone-O-hexoside, Marein, 3',5,5',7-Tetrahydroxy flavanone Okanin, Dicaffeoylquinic acid, Coreopsin.

\section{The changes in food intake and body weight in mice}

Hyperlipidemia mice model intake less food than that in the control group $(\mathrm{P}<0.01)$. Hyperlipidemia mice lost slight weight but showed no significant difference compared with control group. Throughout the experimental period, Xinjiang Coreopsis tinctoria groups showed no significant difference in food taking and body weight compared with hyperlipidemia model group, indicating that total flavonoids from Xinjiang Coreopsis tinctoria has no apparent adverse effects or acute toxicity on mice. The changes in food intake were shown in Table 1 and body weight shown in Table 2 .

\section{Mouse serum TG, TC, HDL, LDL change}

The results are shown in Tables 3 and 4. Throughout the experimental period, TC and TG level in the hyperlipidemia mice are significantly higher than normal diet group $(\mathrm{P}<0.05$ or $\mathrm{P}<0.01)$, indicating the success of the hyperlipidemia model. The total flavonoids from Coreopsis tinctoria extract decreased serum TC and TG 4 weeks after drug administration. The total flavonoids from Coreopsis tinctoria extract have no obvious impact on HDL and LDL or other adverse effects. The serum TC and TG in Fenofibrate group decreased $(\mathrm{P}<0.05)$, showing no statistically significant differences in HDL levels or LDL.

The mouse liver weight, liver weight index, liver TC and TG The liver TC and TG in the hyperlipidemia model, the liver weight, liver weight index were significantly higher than the control group $(\mathrm{P}<0.01)$ as shown in Table 5 . At 6 weeks, total flavonoids from plant extract groups liver $\mathrm{TC}$ and liver weight index decreased $(\mathrm{P}<0.05$ or $\mathrm{P}<0.01)$. Liver TG and liver weight showed no statistically significance. Fenofibrate group decreased TC $(\mathrm{P}<0.01)$, but increased liver weight index and liver weight significantly $(\mathrm{P}<0.01)$, no statistically significant changes in liver TG. The results are shown in Table 5.

\section{Effects of Coreopsis tinctoria on histopathological changes of livers}

Photomicrographs of hepatic specimens stained with $H \& E$ were shown in Figure 2. In normal diet group, the livers showed the normal lobular structure with central veins and radiating hepatic cords, no obvious lipid deposition was found. Model fed with hyperlipid diet for 8 weeks developed a higher degree of steatosis,mostly classified as macrovesicular, with a mean grade more than 2. In CT100 mg/kg, CT200mg/kg and Fenofibrate groups, much smaller microvesicular steatosis was seen in Figure 2. The groups had much less and lower size vacuoles compared with the model group (Figure 2). Results showed that hepatic ADRP protein expressions

Table 4 The blood lipid levels at 6 weeks after drug administration

\begin{tabular}{llcccc}
\hline & $\mathbf{n}$ & TC $(\mathbf{m m o l} / \mathbf{L})$ & TG $(\mathbf{m m o l} / \mathbf{L})$ & HDL $(\mathbf{m m o l} / \mathbf{L})$ & LDL $(\mathbf{m m o l} / \mathbf{L})$ \\
\hline Normal diet & 11 & $3.27 \pm 0.34^{* *}$ & $1.65 \pm 0.27^{* *}$ & $2.52 \pm 0.21$ & $0.05 \pm 0.06^{*}$ \\
Model & 12 & $4.17 \pm 0.70$ & $2.69 \pm 1.15$ & $2.67 \pm 0.34$ & $0.43 \pm 0.45$ \\
CT $100 \mathrm{mg} / \mathrm{kg}$ & 12 & $3.91 \pm 0.45$ & $1.66 \pm 0.55^{*}$ & $2.48 \pm 0.19$ & $0.67 \pm 0.27$ \\
CT $200 \mathrm{mg} / \mathrm{kg}$ & 12 & $3.88 \pm 0.47$ & $1.54 \pm 0.48^{* *}$ & $2.40 \pm 0.17^{*}$ & $0.78 \pm 0.30$ \\
Fenofibrate & 12 & $3.62 \pm 0.62^{*}$ & $1.92 \pm 0.42^{*}$ & $2.51 \pm 0.26$ & $0.25 \pm 0.15$ \\
\hline
\end{tabular}

Compared with hyperlipidemia control, ${ }^{*} \mathrm{P}<0.05$; ${ }^{* *} \mathrm{P}<0.01$. 
Table 5 Liver TG, Liver weight and liver weight index at 6 weeks after drug administration

\begin{tabular}{llcccc}
\hline & $\mathbf{n}$ & TC $(\mathbf{m g} / \mathbf{g}$ wet tissue) & TG (mg/g wet tissue) & Liver weight $\mathbf{g})$ & liver weight index $\mathbf{g} / \mathbf{1 0 0} \mathbf{g}$ weight) \\
\hline Normal diet & 11 & $7.08 \pm 0.48^{* *}$ & $29.08 \pm 5.73^{* *}$ & $1.80 \pm 0.16^{* *}$ & $3.72 \pm 0.31^{* *}$ \\
Model & 12 & $16.33 \pm 5.47$ & $76.88 \pm 20.04$ & $2.46 \pm 0.27$ & $5.24 \pm 0.33$ \\
CT $100 \mathrm{mg} / \mathrm{kg}$ & 12 & $11.61 \pm 1.39^{* *}$ & $63.67 \pm 9.59$ & $2.30 \pm 0.30$ & $4.86 \pm 0.46^{*}$ \\
CT $200 \mathrm{mg} / \mathrm{kg}$ & 12 & $12.53 \pm 1.84^{*}$ & $66.31 \pm 11.51$ & $2.31 \pm 0.22$ & $4.96 \pm 0.35^{*}$ \\
Fenofibrate & 12 & $11.30 \pm 1.72^{* *}$ & $77.43 \pm 11.48$ & $4.21 \pm 0.64^{* *}$ & $9.12 \pm 0.93^{* *}$ \\
\hline
\end{tabular}

Compared with hyperlipidemia model, ${ }^{*} \mathrm{P}<0.05$; ${ }^{* *} \mathrm{P}<0.01$.

were suppressed in flavonoid-treated groups as seen in Figure 3.

ALT and AST are the marker of liver function. In the hyperlipidemia mice group, ALT, AST and ALP showed no significant increase seen in Table 6. After given low and high dose flavonoids from Coreopsis tinctoria extract, the ALT, AST and ALP did not change significantly either. Wihile in Fenofibrate group ALT, AST, ALP increased significantly (ALT, AST, P $<0.05$; ALP, $\mathrm{P}<0.01$ ) (seen in Table 6).

\section{Discussion}

In this study we investigate the active ingredient identification, biomedical function and molecular mechanism of Coreopsis tinctoria on lipid. An efficient method for enrichment of flavonoids from the flowers of Coreopsis tinctoria was developed using macroporous resin. HPLC proved that flavonoids are functional ingredients abundant in Coreopsis tinctoria. Flavonoids reduce TC and TG level in hyperlipidemiaadipose mice by down regulating adipose differentiation-related protein (ADRP) expression.

Flavonoids are polyphenolic compounds produced by plants and proved effective in prevention of cardiovascular, carcinogenic, neurodegenerative and immune diseases. Here we investigate its effect on modulating the lipid metabolism. Hyperlipidemia is a lipid metabolic disease due to abnormal fat metabolism [8]. In this study, we use high fat diet by intragastric administration to set up a hyperlipidemia model in mice. The serum TC and TG, LDL levels were higher than those in the control group $(\mathrm{P}<0.05$, $\mathrm{P}<0.01$ ), indicating successful establishment of mouse model of hyperlipidemia. Treatment with flavonoids from Coreopsis tinctoria of low and high dose groups can decrease serum TG in hyperlipidemia animal, and there is a clear dose-response relationship.

Studies have shown that lipid metabolism disorder and insulin resistance lead to liver lipid and triglycerides production increased, exceeding its transfer capacity, causing lipid accumulation in liver, or even liver damage [9]. The results of this study showed in the liver tissue of hyperlipidemia mouse model, the TC, TG, liver weight and liver weight index were higher than those in the control group. Compare with hyperlipidemia model, the low and high dose of total flavonoids can decrease the TC and liver weight indexes, indicating that total flavonoids from Coreopsis tinctoria extract can not only reduce blood fat, but

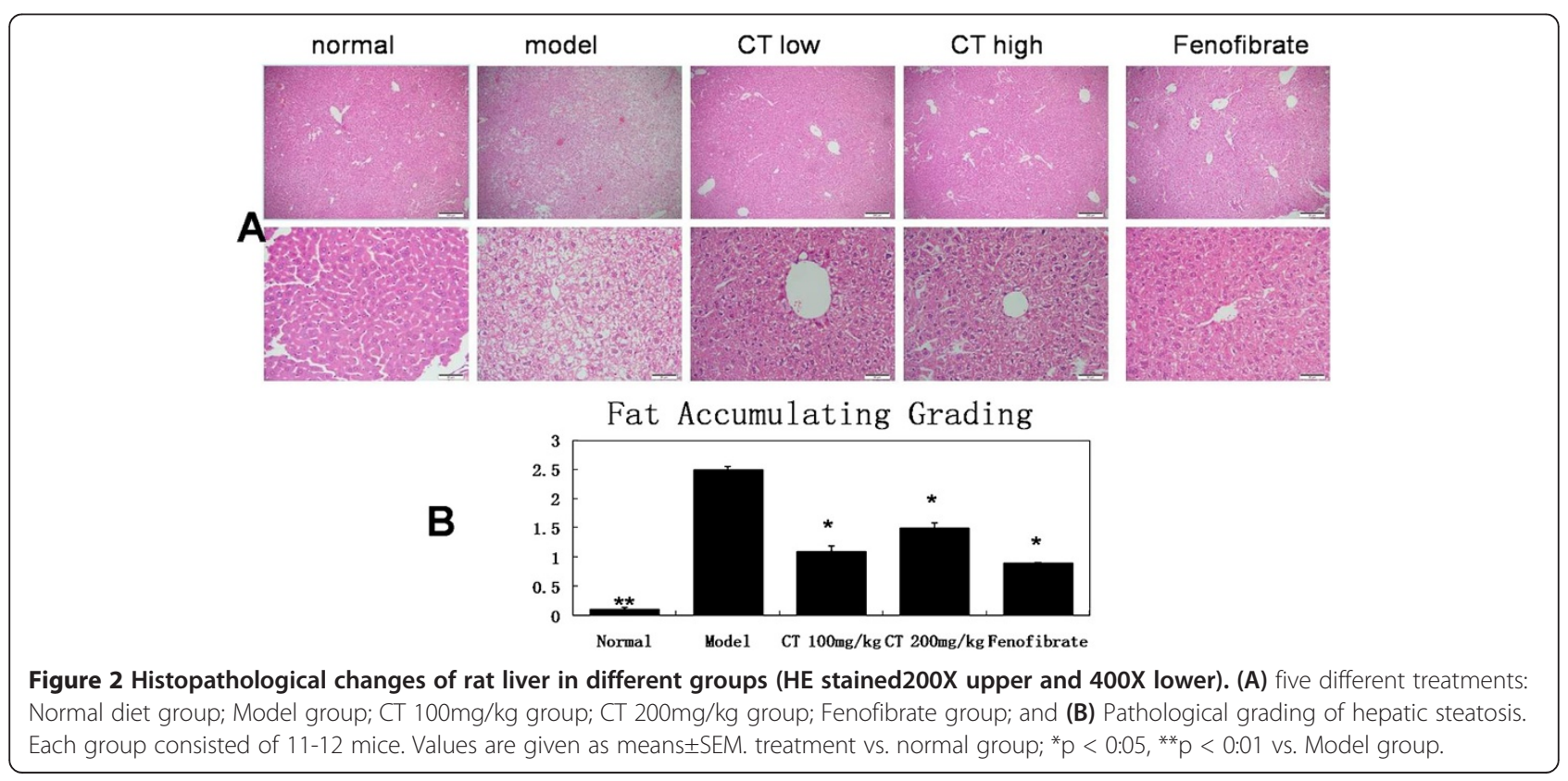



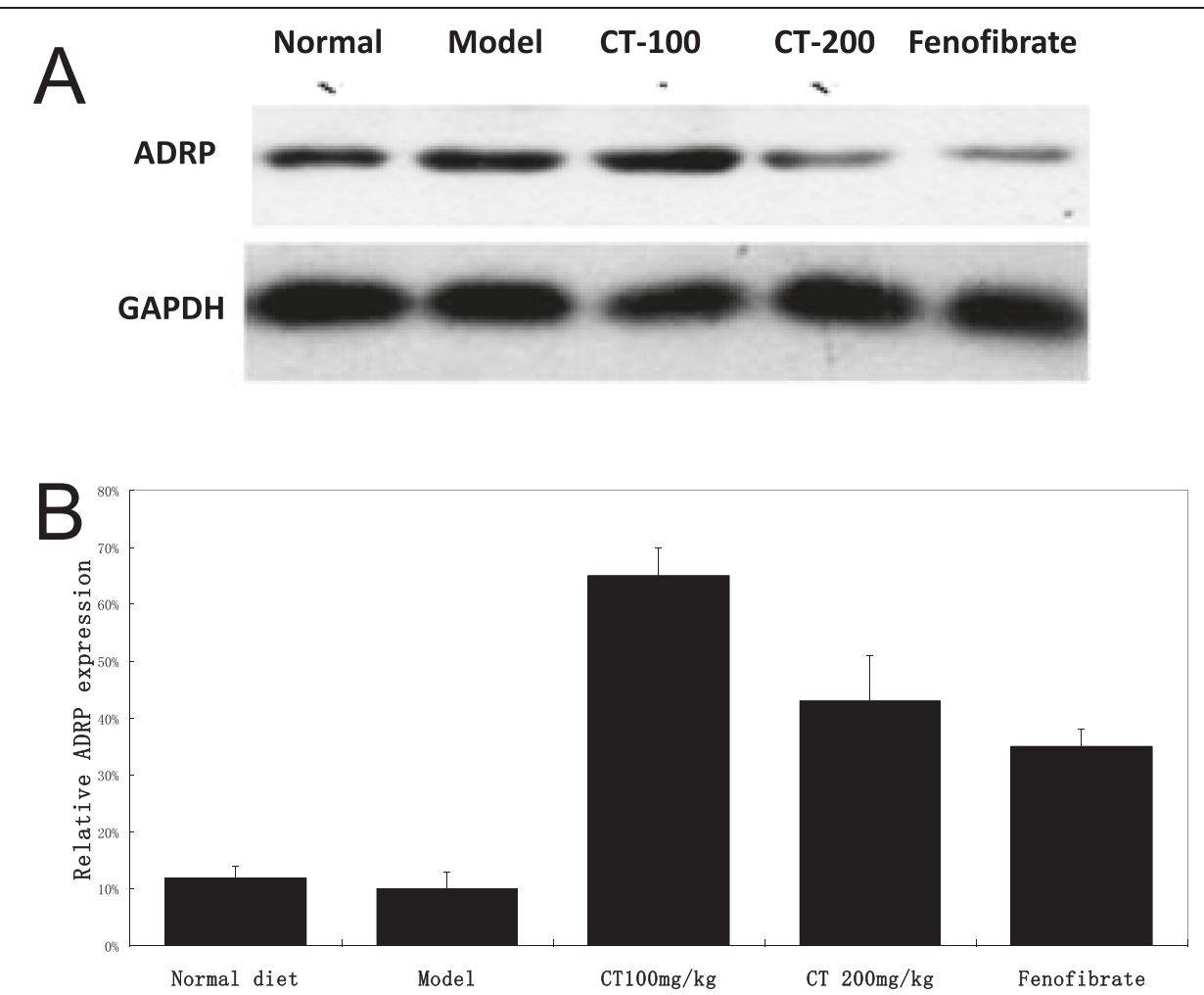

Figure 3 Western blotting for ADRP expression in livers. Representative Western blot of ADRP (A) as well as quantitative analysis of blots (B). ADRP protein level corrected by GAPDH. Data are presented as mean _ SEM. ${ }^{*} p<0: 05,{ }^{* *} p<0: 01$ vs. Normal group.

also reduce the liver lipid, protecting liver function. There are chemical drugs for hyperlipidemia treatment among which Fenofibrate is a commonly used medicine. The long time use of Fenofibrate caused side effects such as liver damage and muscle breakdown [10]. In the Fenofibrate group the mice serum TC and liver tissue TG decrease, serum LDL also decreased, but it increased ALP, ALT and AST.

ALT and AST are the main index reflecting liver function status. ALT exists in the liver cells, AST exists in mitochondrial and cytosolic. Alkaline phosphatase ALP is widely distributed in many organs such as the liver, bones, intestines, kidneys and the placenta. Enzymes in the liver cells and liver sinusoids have a concentration gradient. In

Table 6 AST, ALT and ALP at 6 weeks after drug administration

\begin{tabular}{llccc}
\hline & $\mathbf{n}$ & ALT (u/L) & AST (u/L) & ALP (u/L) \\
\hline Normal diet & 11 & $51.88 \pm 17.99$ & $113.87 \pm 6.12$ & $101.28 \pm 2.91$ \\
Model & 12 & $53.28 \pm 12.12$ & $93.72 \pm 6.44$ & $105.27 \pm 3.12$ \\
CT $100 \mathrm{mg} / \mathrm{kg}$ & 12 & $54.12 \pm 13.74$ & $103.45 \pm 7.32$ & $102.12 \pm 3.22$ \\
CT $200 \mathrm{mg} / \mathrm{kg}$ & 12 & $61.24 \pm 19.82$ & $121 \pm 8.91$ & $104.21 \pm 2.87$ \\
Fenofibrate & 12 & $78.87 \pm 18.12^{*}$ & $131.49 \pm 6.21^{*}$ & $412.21 \pm 21.2^{* *}$ \\
\hline
\end{tabular}

Compared with normal diet control, ${ }^{*} \mathrm{P}<0.05 ; * * \mathrm{P}<0.01$. the case of oxidative injury, liver cell membranes were attacked by free radical and the permeability changes, leading to markedly elevated serum ALT, ALP with the involvement of mitochondria, the endoplasmic reticulum membrane system [11]. However the hyperlipidemia mice showed no ALT, AST or ALP increase, indicating the hyperlipemia is not high enough to cause changes in ALT, AST and ALP. The low and high dose groups of using total flavonoids from Coreopsis tinctoria extract, the ALT, AST and ALP do not change significantly. As a comparison, Fenofibrate group had significant ALT, AST, ALP increase, suggesting that Fenofibrate has damage in liver function. The total flavonoids of Coreopsis tinctoria extracts of liver damage has less impact on liver function.

Western-blot showed that adipose differentiation-related protein (ADRP) protein expression was higher in model model group. ADRP is expressed in the liver and its expression increases in the early stage of adipose differentiation or lipid accumulation in hepatocytes [12]. ADRP causes expansion of the number and size of lipid droplets in the liver, which results in lipid accumulation and insulin signaling inhibition. ADRP absence prevented the development of alcoholic fatty liver, associated with a reduction in the levels of triglycerides in the liver. The level of ADRP protein expression is a reliable marker for liver steatosis [12]. The present results showed that hepatic ADRP 
protein expressions were suppressed in flavonoid-treated groups as seen in Figure 3.

Coreopsis tinctoria Nutt.have been used traditionally in Portugal to control hyperglycaemia and a study revealed that daily administration of Coreopsis tinctoria Nutt promoted the recovery of glucose tolerance by inhibition of glucose absorption and direct promotion of insulin secretion. Dias T et al. [5] found that the bioactivity of several flavonoids in Coreopsis tinctoria extracts promote pancreatic cell function recovery through a mechanism of action other than merely antioxidant mediated. Our result confirmed that flavonoids in Coreopsis tinctoria Nutt work as bioactive metabolites with antihyperglycaemic and antihyperlipemia function.

\section{Conclusion}

In summary, flavonoids from Coreopsis tinctoria extracts showed anti- hyperlipidemia effect, particularly in lowering triglycerides, reducing lipid deposition and protecting the liver function. The mechanism of protective effects on hepatic steatosis and liver functions is associated with regulating lipid metabolism through down-regulating blood fat via suppression ADRP expression. The experimental results provide the biomedical experiment basis for using total flavonoids from Coreopsis tinctoria extract to treat hyperlipidemia.

\section{Competing interests}

The authors declare that they have no competing interests.

\section{Authors' contributions}

MW and $J L$ conceived and designed the experiments; XC and JX performed the experiments; $Y L$ and $X C$ wrote the manuscript. All authors read and approved the final manuscript.

\section{Acknowledgements}

The research is supported by National Natural Science Foundation of China (81372425 and 81460634), SRF for ROCS, SEM (No. J20120279), Xinjiang Science and Technology Bureau Project (No.2013911131) and Xinjiang Medical University Natural Science Grant (No. 2013ZRZD07)

\section{Author details \\ 'The Department of Internal Medicine, The First Affiliated Hospital of Xinjiang Medical University Urumqi, Xinjiang 830000, China. ${ }^{2}$ The Department of Pharmacy, Shandong University of Traditional Chinese Medicine, Jinan, Shandong 250014, China. ${ }^{3}$ College of Pharmaceutical Sciences, Soochow University, Suzhou, Jiangsu 215123, China. ${ }^{4}$ The Department of Hepatobiliary and Pancreatic Surgery, The First Affiliated Hospital Zhejiang University School of Medicine, Hangzhou, Zhejiang 310003, China.}

Received: 25 July 2014 Accepted: 30 November 2014 Published: 15 December 2014

\section{References}

1. Del Ben M, Polimeni L, Baratta F, Pastori D, Loffredo L, Angelico F: Modern approach to the clinical management of non-alcoholic fatty liver disease. World J Gastroenterol 2014, 20(26):8341-8350.

2. Scarisbrick JJ, Morris S, Azurdia R, Illidge T, Parry E, Graham-Brown R, Cowan R, Gallop-Evans E, Wachsmuth R, Eagle M, Wierzbicki AS, Soran H, Whittaker S, Wain EM: U.K. consensus statement on safe clinical prescribing of bexarotene for patients with cutaneous T-cell lymphoma. Br J Dermatol 2013, 168(1):192-200
3. Kma L: Plant extracts and plant-derived compounds: promising players in a countermeasure strategy against radiological exposure. Asian Pac $J$ Cancer Prev 2014, 15(6):2405-2425.

4. Sun YH, Zhao J, Jin HT, Cao Y, Ming T, Zhang LL, Hu MY, Hamlati H, Pang SB, Ma XP: Vasorelaxant effects of the extracts and some flavonoid from the buds of Coreopsis tinctoria. Pharm Biol 2013, 51(9):1158-1164.

5. Dias T, Bronze MR, Houghton PJ, Mota-Filipe H, Paulo A: The flavonoid-rich fraction of Coreopsis tinctoria promotes glucose tolerance regain through pancreatic function recovery in streptozotocin-induced glucose-intolerant rats. J Ethnopharmacol 2010, 132(2):483-490.

6. Dias T, Liu B, Jones P, Houghton PJ, Mota-Filipe H, Paulo A: Cytoprotective effect of Coreopsis tinctoria extracts and flavonoids on tBHP and cytokine-induced cell injury in pancreatic MIN6 cells. J Ethnopharmacol 2012, 139(2):485-492. doi:10.1016/j.jep.2011.11.038. Epub 2011 Nov 28.

7. Salvamani S, Gunasekaran B, Shaharuddin NA, Ahmad SA, Shukor MY: Antiartherosclerotic effects of plant flavonoids. Biomed Res Int 2014, 2014:480258.

8. Sharma K, Kumar K, Mishra N: Nanoparticulate carrier system: a novel treatment approach forhyperlipidemia. Drug Deliv 2014, 6:1-16.

9. Berlanga A, Guiu-Jurado E, Porras JA, Auguet T: Molecular pathways in non-alcoholic fatty liver disease. Clin Exp Gastroenterol 2014, 7:221-239.

10. Rull A, Geeraert B, Aragonès $G$, Beltrán-Debón R, Rodríguez-Gallego E, García-Heredia A, Pedro-Botet J, Joven J, Holvoet P, Camps J: Rosiglitazone and fenofibrate exacerbate liver steatosis in a mouse model of obesity and hyperlipidemia. A transcriptomic and metabolomic study. J Proteome Res 2014, 13(3):1731-1743.

11. Tarantino G, Finelli C, Scopacasa F, Pasanisi F, Contaldo F, Capone D, Savastano S: Circulating levels of sirtuin 4, a potential marker of oxidative metabolism, related to coronary artery disease in obese patients suffering from NAFLD, with normal or slightly increased liver enzymes. Oxid Med Cell Longev 2014, 2014:920676.

12. Grasselli E, Voci A, Pesce C, Canesi L, Fugassa E, Gallo G, Vergani L: PAT protein mRNA expression in primary rat hepatocytes: effects of exposure to fatty acids. Int J Mol Med 2010, 25(4):505-512.

doi:10.1186/1476-511X-13-193

Cite this article as: Li et al:: Flavonoids furom Coreopsis tinctoria adjust lipid metabolism in hyperlipidemia animals by down-regulating adipose differentiation-related protein. Lipids in Health and Disease 2014 13:193.

\section{Submit your next manuscript to BioMed Central and take full advantage of:}

- Convenient online submission

- Thorough peer review

- No space constraints or color figure charges

- Immediate publication on acceptance

- Inclusion in PubMed, CAS, Scopus and Google Scholar

- Research which is freely available for redistribution 